\title{
Inflammasomes: too big to miss
}

\author{
Andrea Stutz, ${ }^{1}$ Douglas T. Golenbock, ${ }^{1}$ and Eicke Latz ${ }^{1,2}$ \\ ${ }^{1}$ Department of Infectious Diseases and Immunology, University of Massachusetts Medical School, Worcester, Massachusetts, USA. ${ }^{2}$ Institute of Innate \\ Immunity, University of Bonn, Bonn, Germany.
}

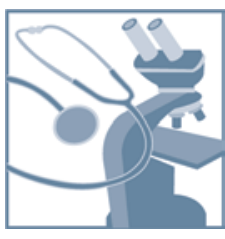

Inflammation is the coordinated immune response to harmful stimuli that appear during infections or after tissue damage. Cells of the innate immune system are the central players in mediating inflammatory tissue responses. These cells are equipped with an array of signaling receptors that detect foreign molecular substances or altered endogenous molecules that appear under situations of stress. This review provides an overview of recent progress in elucidating the molecular mechanisms that lead to inflammatory reactions. We discuss the current knowledge of the mechanisms leading to the activation of cytoplasmic, multimolecular protein complexes, termed "inflammasomes," which regulate the activity of caspase-1 and the maturation and release of IL-1 $\beta$.

\section{Innate immune signaling receptors orchestrate inflammation}

Inflammation is a host response to infectious agents, tissue damage, or cellular irritants such as toxins. Immune cells encountering these noxious agents become activated and release an array of factors leading to the well-known clinical signs of inflammation: rubor, calor, dolor, tumor, and functio laesa. The structured inflammatory reactions within the local tissue lead to the recruitment of a variety of immune cells, which act in concert with local tissue cells to remove the infectious agent, clear damaged tissue components, and initiate tissue repair processes. This inflammatory response has the effect of protecting the host from further tissue damage and restoring tissue function. However, under certain conditions, immune responses themselves can injure tissues or result in chronic inflammatory processes. The inflammatory response is the result of the activity of a plethora of cell-derived mediators (e.g., chemokines, cytokines, antimicrobial peptides, and reactive oxygen and nitrogen species) and of activated biochemical cascades originating in the vascular compartment (e.g., complement, coagulation, and fibrinolytic systems).

How cells respond to infectious and non-infectious inflammatory stimuli has long been of immense interest. Research in the field of inflammation and in particular innate immunity has led to a more detailed, albeit still fragmentary, understanding of the molecular mechanisms by which many innate immune signaling receptors activate cells.

The innate immune system is found in all species of plant and animal life and provides the immediate host immune response to infectious or noxious insults. The innate immune system relies on germline-encoded receptors for the detection of microbial substances or molecules that arise during tissue damage or other sorts of cellular stress.

Several families of innate immune signaling receptors have so far been identified. The subcellular location of these receptors varies substantially and reflects their biological roles. The transmembrane TLRs and C-type lectin receptors are situated on the plasma membrane and on internal membranes (endosome, lysosome, and endoplasmic reticulum). The cytosol contains the retinoic acidinducible gene-I-like helicases (RLRs) and the nucleotide-binding domain leucine-rich repeat-containing receptors (NLRs). Together,

Conflict of interest: The authors have declared that no conflict of interest exists. Citation for this article: J. Clin. Invest. 119:3502-3511 (2009). doi:10.1172/JCI40599. these signaling receptors can detect a broad variety of molecular entities including lipids, proteins, nucleic acids, and combinations thereof. While it is likely that innate immune receptors evolved to recognize specific molecules associated with microbes, many of these same receptors can also detect molecular changes that occur during tissue damage. Ligands for innate immune receptors can be of foreign nature, i.e., not synthesized by the host, such as bacterial LPS. Additionally, the innate immune system can detect molecules that are normally sequestered in specific subcellular compartments and that relocate upon cellular damage, such as the nuclear protein HMGB1. Other emerging principles of innate receptor activation are the ability of the host to detect modified self-molecules that appear under disease conditions, including the response to certain glycosylated proteins or the recognition of crystalline materials, such as may occur in gout (1). Indeed, the scope of the innate immune system is very large, and our understanding of its function is likely to expand exponentially in the next few years. This review focuses on the mechanisms of activation of a subset of signaling receptors of the NLR family of proteins.

\section{NLR proteins and inflammasomes}

NLRs are a family of intracellular immune receptors with more than 20 members currently known in humans. The NLR family members are characterized by the presence of at least two shared features, leucine-rich repeats (LRRs) near the $\mathrm{C}$ terminus and a NACHT nucleotide-binding domain (NBD). The LRR domains of this family are thought to play a role in autoregulation, the recognition of pathogen-associated molecular patterns (PAMPs), and/or protein-protein interactions. The NBDs can bind ribonucleotides, possibly regulating self-oligomerization $(2,3)$.

Although the NLRs share common features, they differ in their N-terminal domains. These differences are used to further subcategorize the NLR protein members (4). The largest group, comprising 14 members, has an N-terminal pyrin domain (PYD) and is therefore called "NLRP" (previously also called "NALPs"). Another group, which shares an N-terminal caspase recruitment domain (CARD), contains the proteins nucleotide-binding oligomerization domain-containing 1 (NOD1, also known as NLRC1), NOD2 (NLRC2), as well as NLR family, CARD domain-containing 4 (NLRC4, also known as CARD12 or IPAF). Other NLR family members have an acidic transactivation domain or a baculoviral inhibitory repeat-like domain, such as NLR family, apoptosis inhibitory protein 5 (NAIP5). 
A NLRP1 inflammasome

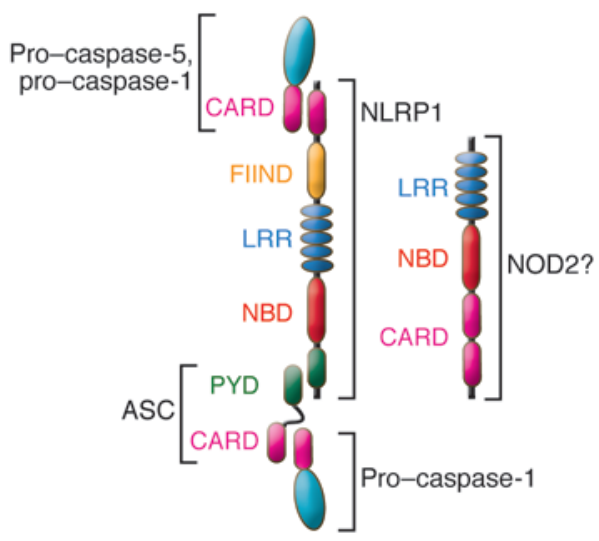

B NLRC4 inflammasome

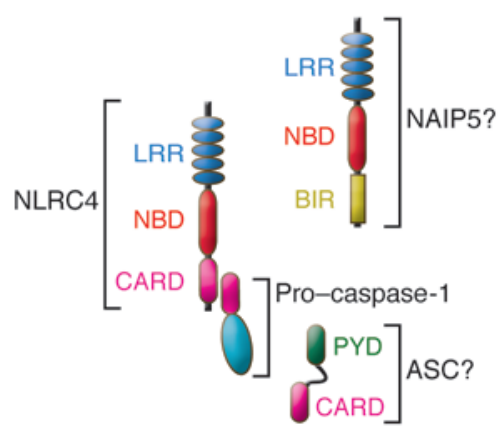

\section{Figure 1}

Graphical depiction of known inflammasomes. (A) NLRP1 contains, in addition to the NLR-typical LRR and NBD domains, a PYD, a FIIND, and a CARD. NLRP1 can recruit pro-caspase- 1 and -5 and possibly forms a complex with NOD2. Recruitment of ASC enhances activation of pro-caspase- 1 . (B) NLRC4 contains a CARD that can directly recruit pro-caspase-1. Reports further demonstrate a role for ASC and possibly NAIP5 in NLRC4 inflammasome activation. (C) NLRP3 activates pro-caspase-1 via recruitment of ASC. (D) AIM2 is a bipartite protein consisting of a PYD and DNA-binding HIN200 domain and recognizes cytoplasmic double-stranded DNA and assembles the DNA inflammasome with ASC and pro-caspase-1.

\section{NLRP3 inflammasome}

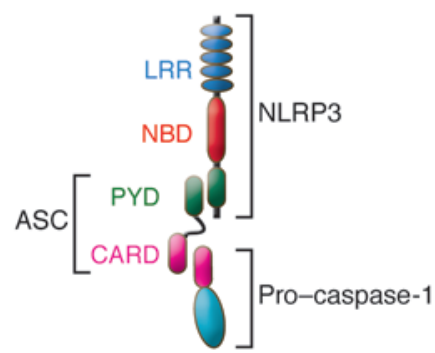

D DNA inflammasome

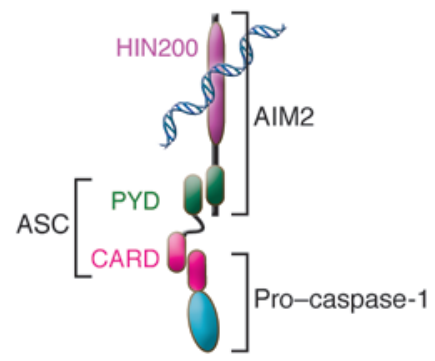

Several members of the NLR family, including NLRP1, NLRP3, and NLRC4 can assemble multimolecular complexes in response to various activators, leading to the activation of inflammatory caspases. Activated caspase- 1 controls the maturation of the cytokines of the IL-1 family. In analogy to the apoptosome, which activates apoptosis-inducing caspases (5-7), the NLR multimolecular complexes are termed "inflammasomes," as they control inflammatory caspases $(8,9)$.

According to the NLRs involved, the inflammasomes are NLRP1, NLRP3, or NLRC4. Recently, the protein absent in melanoma 2 (AIM2, also known as PYHIN4), which belongs to a different protein family (PYHIN), was also reported to assemble an inflammasome (10-13). This review describes the different inflammasomes, their stimuli, and their activation pathways (see Figure 1).

\section{Inflammasome formation leads to caspase-1 activation and maturation of IL-1 family cytokines}

The exact sequence of events leading to inflammasome activation is not well understood for all inflammasomes. It is believed that upon stimulation, the respective inflammasome oligomerizes and recruits pro-caspase- 1 directly via a CARD homotypic interaction (e.g., NLRP1 or NLRC4 inflammasomes) or indirectly via the adaptor protein apoptosis-associated speck-like protein containing a CARD (ASC, or Pycard). In the latter case, NLRP3 or AIM2, for example, interact with ASC via the homotypic interaction of their PYDs. ASC, in turn, interacts with pro-caspase- 1 via their CARDs.

In resting cells, caspase- 1 is present in a catalytically inactive pro-form. The formation of the inflammasome initiates autocatalytic activation of caspase- 1 by mediating the apposition of two or more caspase- 1 monomers, resulting in cleavage of the pro-enzyme into a $20-\mathrm{kDa}(\mathrm{p} 20)$ and a $10-\mathrm{kDa}(\mathrm{p} 10)$ subunit (14). The active enzyme then assembles into two heterodimers of p20 and p10 subunits, containing two active sites (15).

Caspase- 1 was initially known as IL-1-converting enzyme, since its first known substrate, pro-IL-1 $\beta$, is proteolytically converted into the bioactive cytokine, IL-1 $\beta$ (14). Mature IL-1 $\beta$ is a potent pyrogen with pleiotropic functions including the activation of lymphocytes and endothelial cells and the initiation of the acute phase response (16). Due to this repertoire of highly potent proinflammatory activities, accidental release of IL- $1 \beta$ would be quite deleterious, which explains the development of a complicated mechanism of regulation in mammals. The regulation of IL-1 $\beta$ secretion includes priming, maturation of both caspase- 1 and IL-1 $\beta$, and release of the active protein (17).

Caspase- 1 can also cleave other members of the IL-1 family. Caspase-1-mediated cleavage has been shown for pro-IL-18 (18). The proform of this cytokine is constitutively expressed and does not require priming for induction. IL-18 can induce IFN- $\gamma$ and pro-inflammatory cytokine secretion and activates NK cells. Caspase- 1 can also cleave IL-33, another member of the IL-1 family, but in contrast to the other mentioned cytokines, IL-33 is inactivated by caspase-1-mediated processing and activated by another enzyme, calpain (19-21).

The mechanisms leading to secretion of the mature cytokines are not well understood. Several models for their export have been proposed, such as secretion via multivesicular bodies containing exosomes, via shedding of microvesicles from the plasma membrane or directly through the plasma membrane via unidentified transporters (22-25).

Under certain circumstances, release of the cytokines precedes or is concomitant with a caspase-1-induced inflammatory cell death 
called "pyroptosis". Recent reviews on cell death in response to infection describe the morphology and significance of pyroptosis in more detail $(26,27)$. In brief, pyroptosis shows characteristics of both apoptosis and necrosis. It is a rapid process involving caspase-1-dependent DNA fragmentation and pore formation, which leads to cellular lysis (28). Other effector mechanisms downstream of caspase- 1 are currently under investigation. For example, caspase- 1 was reported to inhibit the glycolysis pathway by cleaving key enzymes, including aldolase and glyceraldehyde-3-phosphate dehydrogenase (29). This process and cleavage of the apoptosis effector caspase-7 by caspase-1 (30) might be starting points for this special form of cell death.

However, cell death is not found in all cases of caspase- 1 activation. In response to pore-forming toxins (via the NLRP3 inflammasome; see below), caspase- 1 can promote cell survival by activating lipid biosynthesis in Chinese hamster ovarian cells and HeLa cells (31). This suggests a possible switch between cell death and repair after activation of caspase-1. A cell type-specific regulation might be an alternative explanation. A recent report suggests that caspase- 1 is not only important for cleavage of substrates, but also regulates unconventional protein secretion. Caspase- 1 influences the release of many proteins without signal sequences, including pro-IL-1 $\alpha$ and other mediators involved in tissue repair (32).

Components and stimuli of the different inflammasomes The NLRP1 inflammasome. NLRP1 (also known as NALP1) differs from the other members of the NLR family in its domain organization. Like all members of the NLRP subgroup, it has an N-terminal PYD, followed by an NBD and an LRR region. However, in contrast to all other members, NLRP1 has a C-terminal extension consisting of a FIIND motif and a CARD. One of its first identified interaction partners was ASC, to which it binds via homotypic PYD-PYD interactions (33). In 2002, the identification of a caspase-1-activating multimolecular complex consisting of caspase-1, caspase-5, ASC, and NLRP1 was reported and termed "inflammasome" (8), a name that has endured through the intervening years. The mechanisms of NLRP1 activation were further elucidated by Reed and colleagues, who showed in a cell-free system that the minimal components of this inflammasome were NLRP1, caspase-1, and a triphosphate ribonucleotide (NTP). However, assembly was enhanced with ASC (34). They also discovered muramyl dipeptide (MDP) as an activating ligand and suggested a two-step mechanism of activation of NLRP1. First, MDP binds to NLRP1, inducing a conformational change, which allows subsequent binding of the NTP and self-oligomerization of NLRP1 (34). In resting cells, the anti-apoptotic proteins Bcl-2 and Bcl-X(L) bind NLRP1, thereby suppressing caspase- 1 activation and IL- $1 \beta$ production. In activated macrophages, NLRP1 is released from Bcl-2 and $\mathrm{Bcl}-\mathrm{X}(\mathrm{L})$ and can interact with its ligands $(35,36)$. These studies demonstrate how apoptotic pathways and inflammasome activation are tightly linked. Another stimulus for NLRP1 is cytosolic anthrax lethal toxin (LT), but this has only been shown for murine Nlrp1b. The exact mechanism remains unclear, as caspase-1 activation depends on the proteolytic activity of LT and on proteasome activity, so there is a possibility that a degraded cellular protein serves as a ligand for the NLRP1 inflammasome or that inhibitory proteins require proteasome-mediated degradation before NLRP1 can be fully activated (37-40).

Recent work also suggests a role for NOD2 in the assembly of the NLRP1 inflammasome $(41,42)$. NOD2 is another member of the NLR family and contains two N-terminal CARDs. Even though in a cell-free system NLRP1 is sufficient to activate caspase- 1 in response to MDP, NOD2 is needed for in vitro sensing of both MDP and LT, and NOD2 can directly interact with pro-caspase-1 and NLRP1, but not NLRP3 (41). NOD2 is a known sensor of MDP and has also been shown to activate NF-кB $(43,44)$. This raises the interesting possibility that MDP could generate both signals necessary for IL- $1 \beta$ production, that is priming via NF- $\kappa B$ and maturation via caspase-1.

The NLRP3 inflammasome. NLRP3 (previously called "NALP3," "PYPAF1," or "cryopyrin") contains the NLR-typical elements (LRR and NBD) and an N-terminal PYD. Like NLRP1, it can recruit the adaptor protein ASC by PYD interactions (45). Oligomerization of NLRP3 in response to a stimulus and subsequent recruitment of ASC can activate caspase-1 (46). In order to oligomerize, NLRP3 further requires binding of ATP or deoxyATP to its NBD element, which has ATPase activity, possibly regulating disassembly (47). Similarly to NLRP1, oligomerization of NLRP3 is inhibited by high $\mathrm{K}^{+}$concentrations (48). Interestingly, only one large NLRP3 inflammasome, up to $2 \mu \mathrm{m}$ in diameter, is formed per cell. This large complex consists mainly of the adaptor ASC (49).

Many stimuli that trigger assembly of the NLRP3 inflammasome have been described. However, the underlying mechanism of activation is poorly understood. The reported stimuli are of diverse physico-chemical natures and include many microbes (viruses, bacteria, and fungi), a range of PAMPs, bacterial toxins, and aggregated substances. The discovery of such a broad range of stimuli has prompted the development of several different hypotheses on the molecular mechanisms that could potentially explain NLRP3 activation. Live bacteria and viruses were reported to activate the NLRP3 inflammasome, including Sendai virus, influenza A virus and adenoviruses, Staphylococcus aureus, Listeria monocytogenes, E. coli, Mycobacterium marinum, and Neisseria gonorrboeae $(48,50-55)$. Several recent reports also link IL-1 $\beta$ production in response to the fungus Candida albicans to the NLRP3 inflammasome (56-58). Microbes express many products that can activate innate immune receptors such as TLRs, RLRs, or other NLRs. In fact, many microbe-derived substances such as MDP, bacterial RNA, LPS, Pam2CysK4, poly(I:C), as well as the pro-inflammatory imidazoquinoline compounds $\mathrm{R} 837$ and $\mathrm{R} 848$, were all reported to activate the NLRP3 inflammasome when administered in the presence of $\operatorname{ATP}(50,59,60)$. In addition, a number of bacterial toxins can activate the NLRP3 inflammasome, including nigericin (from Streptomyces hygroscopicus), listeriolysin O (L. monocytogenes), aerolysin (Aeromonas), and maitotoxin (Gambierdiscus toxicus) $(31,61)$. In addition, $\alpha$-toxin, and $\beta$ - and $\gamma$-hemolysins (S. aureus) can also activate caspase- 1 in the presence of bacterial lipoproteins (62). All of these toxins are pore formers leading to $\mathrm{K}^{+}$efflux from infected cells and can potentially influence lysosomal stability, one condition necessary for NLRP3 inflammasome activation. Interestingly, the IL- $1 \beta$ secretion after exposure to nigericin and maitotoxin (and possibly also other toxins) depends on the presence of pannexin-1, a hemichannel, which forms large pores in the plasma membrane and acidifies endosomal compartments (63-66). However, the role of pannexin- 1 in the activation of the NLRP 3 inflammasome is unclear and has resulted in multiple theories.

In addition to these pathogen-associated stimuli, a number of endogenous stress signals have been reported to activate the NLRP3 inflammasome. The first danger-associated signal described was ATP (61). ATP has a high intracellular concentration 


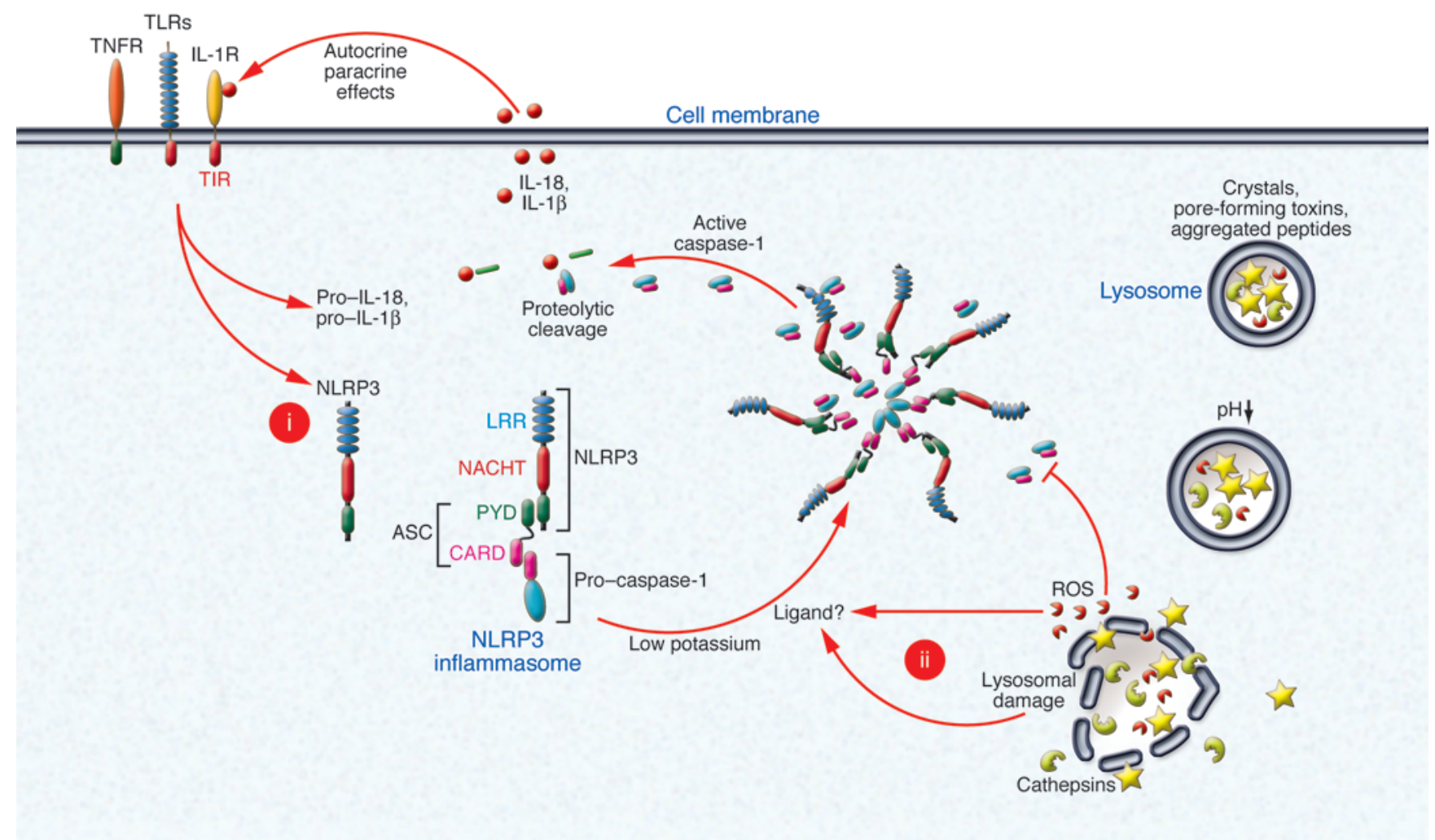

\section{Figure 2}

Inflammasomes form large multimolecular complexes that control the activity of caspase-1. (i) Activation of cytokine receptors or pattern recognition receptors such as TLRs leads to the induction of pro-IL-1 $\beta$ and NLRP3. (ii) In a second step, NLRP3 inflammasome assembly is triggered by low intracellular potassium levels and the binding of a putative ligand that is generated by proteolytic activity after lysosomal damage or by the action of ROS. The assembled NLRP3 inflammasome results in activation of caspase-1, which proteolytically activates IL-1 $\beta$ family cytokines. The produced pro-inflammatory IL-1 $\beta$ family cytokines can act on other cell types or act in a feed-forward loop. High intracellular oxygen tension can also lead to direct deactivation of caspase- 1.

and is kept low in the extracellular space by the activity of ATPases. ATP is released from dying cells, and it is likely that ATP is present locally in abundant quantities in stressed tissues. Stimulated cytotoxic lymphocytes as well as microglia and human monocytes have been shown to release ATP (67-70), and other cell types undoubtedly do as well. While the paradigm for inflammasome activation that we describe above involves a priming step (that results in the production of pro-IL-1 $\beta$ and upregulates NLRP3) and an inflammasome activation step, human monocytes appear capable of producing and processing IL- $1 \beta$ in a combined fashion. The priming of monocytes with PAMPs releases ATP, which serves as the signal for the assembly of the NLRP3 inflammasome and thereby maturation of pro-IL-1 $\beta(57,70)$. Binding of extracellular ATP to the purinergic receptor $\mathrm{P} 2 \mathrm{X} 7$ leads to IL-1 $\beta$ maturation via the effects of purinergic stimulation on the hemichannel pannexin-1 $(63,65)$. Another endogenous "danger" signal that activates the NLRP3 inflammasome is hyaluronan, a structural component of the extracellular matrix (71).

Perhaps the most important function of NLRP3 is its ability to recognize when normally soluble endogenous molecules form crystals. The first crystal-induced inflammatory diseases linked to the NLRP3 inflammasome were gout and pseudogout. Uric acid assembles in the joints of the hyperuricemic patients, forming monosodium urate (MSU) crystals; a similar syndrome of less clear etiology is pseudogout, where calcium pyrophosphate dehydrate crystals are formed and may cause acute arthritis (72). Both of these crystals were shown to activate the NLRP3 inflammasome (73), thus defining the mechanism by which these crystals most likely cause inflammatory arthritis. Similar to the effects of these endogenously generated crystals, several environmental pollutants that consist of inorganic crystalline material have been associated with inflammatory disease. For example, inhalation of asbestos or silica particles can result in pneumonitis, pulmonary fibrosis, and lung cancer. Both crystals are recognized by the NLRP3 inflammasome (74-76). Finally, the proinflammatory activity of crystalline materials has long been exploited as an adjuvant for vaccines. Alum, the most commonly used vaccine adjuvant, triggers caspase-1 via the NLRP3 inflammasome (74, 77-81). Similarly, other particulate vaccines such as poly(lactide-co-glycolide) and polystyrene microparticles induce IL- $1 \beta$ secretion in an NLRP3dependent manner (82). Also, crystals formed by pathogens can be recognized, as was shown for malarial hemozoin (83). In addition to crystals and particulates, peptide aggregates can cause NLRP3 activation. The importance of this observation relates primarily to Alzheimer disease, where extracellular $\beta$-amyloid aggregates are found in disease-associated senile plaques. These aggregates appear to be sensed in the CNS by microglia via NLRP3, leading to the secretion of pro-inflammatory cytokines and nitric oxide 
and, possibly, neuronal cell death (84). Since a number of neurodegenerative diseases are associated with aggregated peptides or proteins, it is conceivable that aggregate-induced inflammation could contribute to the pathology of neuronal damage more generally.

Other danger-associated stimuli with NLRP3-stimulating activity include the skin irritant trinitrochlorobenzene $(85,86)$. Also, UVB irradiation was reported to activate NLRP3-dependent IL-1 $\beta$ secretion (87).

Indeed, one of the features of the NRLP3 inflammasome is the large array of activating molecules. This pleiotropism suggests that NRLP3 activation is likely to be indirect and very complex.

Potential mechanisms of NLRP3 inflammasome activation. There are several theories about the mechanism of NLRP3 inflammasome activation. Apparently conflicting data have been published regarding specific NLRP3 activators. One theory for the mechanism of NLRP3 activation is that low intracellular potassium concentration is the main stimulus. There seems to be a need for $\mathrm{K}^{+}$efflux for the activity of most, if not all, NLRP3 stimuli including poreforming toxins and crystalline activators (48, 75, 88-90). Potassium efflux can be achieved due to the activity of a few stimuli themselves. For example, pore-forming toxins can lead to $\mathrm{K}^{+}$efflux and ATP acts on the P2X7 receptor and pannexin-1, thereby triggering $\mathrm{K}^{+}$efflux (88-90). In human monocytes, a variety of activators can trigger the exocytosis of ATP, which may explain the ability of TLR ligands to activate IL- $1 \beta$ maturation and release $(63,64,69,75)$ in the absence of a second stimulus (compared with the requirement for two signals in mouse macrophage inflammasome activation) $(91,92)$. Low intracellular $\mathrm{K}^{+}$concentrations are required for full assembly of the pyroptosome, a multimeric complex formed of ASC dimers in response to inflammasome activators (49). However, a drop of intracellular $\mathrm{K}^{+}$concentration without the assembly of the NLRP3 inflammasome also regulates other processes. This is the case for the NLRP1 inflammasome (48) as well as for the mitochondrial pathway of apoptosis, where $\mathrm{K}^{+}$efflux facilitates caspase- 9 activation but is not the primary stimulus (reviewed in ref. 93). Physiologic $\mathrm{K}^{+}$concentration almost fully inhibits activation of apoptosis (94). It might therefore be speculated that $\mathrm{K}^{+}$ dependence is a safeguard mechanism to control the formation of caspase-activating complexes, but it is not the sole activation mechanism of inflammasome formation.

In addition to $\mathrm{K}^{+}$efflux, other mechanisms might control the assembly of the NLRP3 inflammasome. There are several published theories, all of which propose a role for different stimuli in addition to $\mathrm{K}^{+}$efflux (see Figure 2 ). One theory postulates a direct ligand-receptor interaction. NLRP3 has N-terminal LRRs, which are utilized by other receptors (e.g., TLRs) to directly bind to microbial products or other stimuli. Ligand-receptor interaction conceptually could result in a conformational change, allowing for oligomerization and inflammasome formation. Indeed, such a mechanism appears to operate in the activation of NLRP1 (34). According to this direct ligand recognition hypothesis, pores formed by pannexin-1 in response to ATP might deliver activators directly into the cytosol, where they bind to NLRP3 or other upstream factors (59). Indeed, MDP is released from acidified endosomal compartments into the cytosol upon ATP stimulation requiring pannexin-1 (95). However, it remains unclear whether one sensor can bind to all the reported ligands, given their difference in structure and shape.

It has also been proposed that ROS act on a target upstream of the NLRP3 inflammasome and indirectly cause its activation
$(48,75,76,96)$. ROS inhibition can reduce the amount of mature IL-1 $\beta$ after stimulation with ATP, R837, MSU crystals, asbestos, and silica $(48,75,76,96)$. RNAi-mediated knockdown of components of NADPH oxidase in THP-1 cells (a human acute monocytic leukemia cell line) inhibited IL-1 $\beta$ production in response to crystals (75). However, the pathway connecting ROS to the inflammasome remains largely unknown, and a study utilizing macrophages lacking functional phagosomal NADPH oxidase did not show a connection to NLRP3 inflammasome activation (74). Nevertheless, a shift of the redox equilibrium by ROS was suggested to lead to PI3K-dependent activation of PKB (Akt), which in turn phosphorylates ERK1/2 (MAPK) (96). Another study suggested that inhibition of HMG-CoA reductase or general dysregulation of isoprenoid biosynthesis triggers Rac1, resulting in PI3K- and PKB-dependent activation of caspase-1 (97). However, the link from MAPK or PKB to the NLRP3 inflammasome remains unknown. Furthermore, a recent study suggested that ROS can in fact inhibit caspase- 1 directly by oxidation and glutathionylation (98). Reduction of ROS by SOD 1 was needed in order to produce mature IL-1 $\beta$ (98).

Another hypothesis is based on the observation that NLRP3activating crystals lead to phagosomal destabilization and that pharmacological disruption of lysosomes could also lead to the activation of the NLRP3 inflammasome even in the absence of crystalline material (74). According to this theory, NLRP3 can sense phagosomal or lysosomal disruption as a danger signal and thereby indirectly sense excessive crystal phagocytosis or escape of microbes from endo-lysosomal compartments into the cytosol. The exact nature of the ligand formed after lysosomal damage remains to be determined. In theory, lysosomal contents could be a danger signal sensed by NLRP3, or lysosomal proteases that have gained access to the cytoplasm could cleave a substrate, which then serves as a ligand for NLRP3. Inhibition of the lysosomal protease cathepsin B led to a substantial, albeit partial decrease of IL-1 $\beta$ maturation in a number of studies, and cathepsin B-deficient macrophages show partially reduced IL- $1 \beta$ secretion upon NLRP3 activation $(53-55,74,82,99)$. In addition, the activation of pannexin-1 pores mentioned above might not only induce $\mathrm{K}^{+}$efflux or grant microbial products access to the cytoplasm, but also destabilize lysosomes and lead to release of lysosomal content into the cytoplasm. In fact, cathepsin B translocation to the cytoplasm has been shown following treatment with the pore-forming toxin nigericin, where caspase-1 activation was dependent on cathepsin B activity (99). However, a cathepsin B knockout did not result in inhibition of caspase- 1 activation after stimulation with hemozoin and C. albicans $(56,83)$. Furthermore, a cathepsin B inhibitor was recently also shown to affect caspase- 1 activation by anthrax LT (possibly via NLRP1b) (100). It thus appears that functional redundancies might exist between different proteases. In addition, cathepsin inhibitors might also affect other inflammasomes.

While the theories for the different modes of NLRP3 inflammasome activation are seemingly different, they are not mutually exclusive. It is possible that ROS generation contributes to lysosomal destabilization (101-104) and that bacterial products indirectly contribute to NLRP3 activation. It is also conceivable that the activation of NLRP3 is a multistep process that requires more than one factor.

In fact, recent studies have demonstrated that priming via transcriptionally active pattern recognition receptors (e.g., TLRs or NODs) or via cytokines is required for subsequent activation of NLRP3 inflammasome by ATP, pore-forming toxins, or crystals in macrophages $(91,92)$. These studies revealed that cell stimulation 


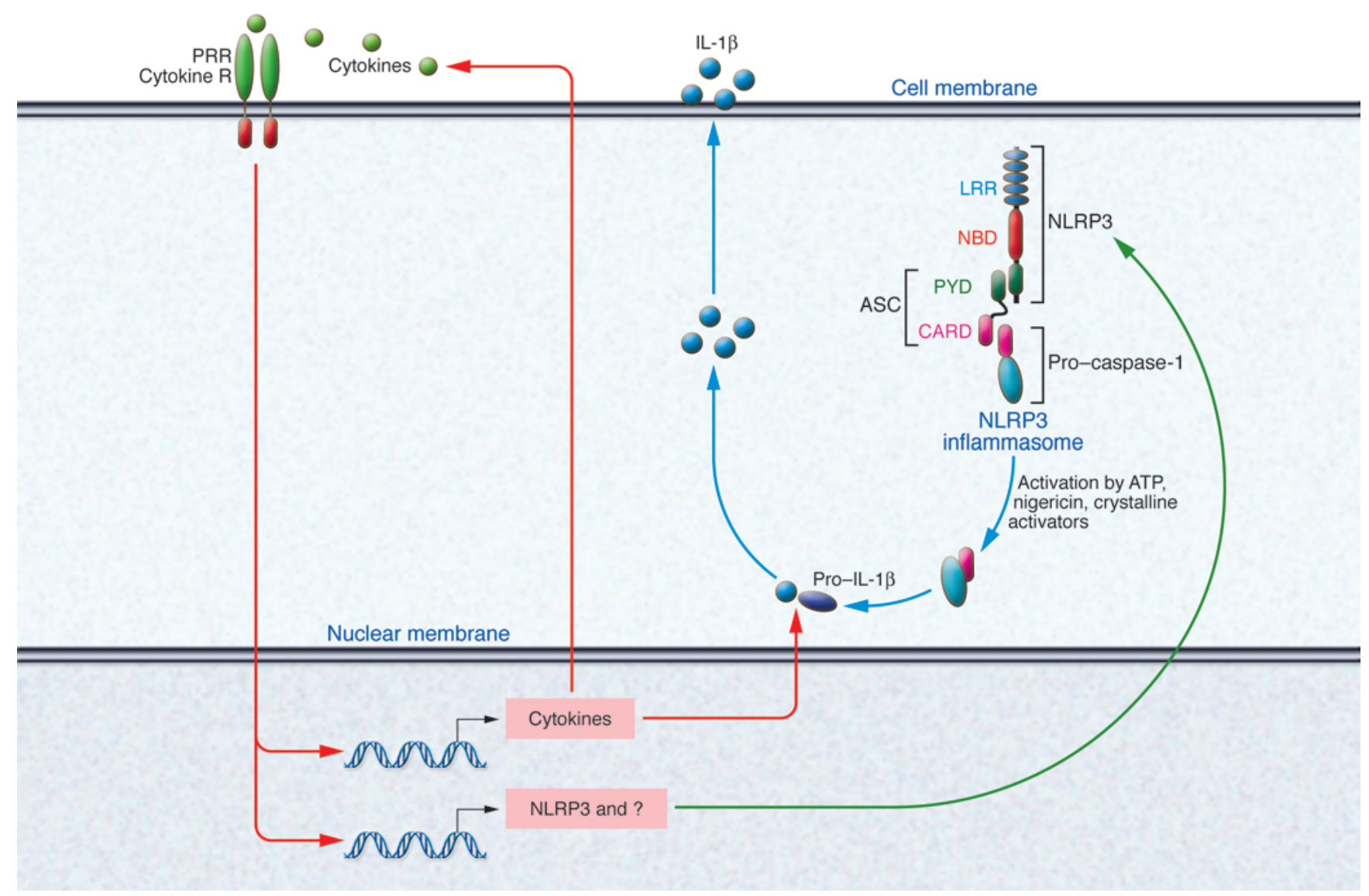

Figure 3

Priming is essential for the activation of the NLRP3 inflammasome and IL-1 $\beta$ secretion. PRR and cytokine receptors $(R)$ activate the expression of the pro-forms of the IL-1 cytokine family and other cytokines. In addition, NLRP3 and possibly other proteins needed for IL-1 $\beta$ secretion are induced. NLRP3 upregulation is required for its activation by ATP, pore-forming toxins, or crystals.

via PRRs or cytokine receptors leads to increased cytosolic NLRP3 levels. If a critical NLRP3 concentration was reached, NLRP3 inflammasome assembly was induced by stimulation with ATP, nigericin, or crystalline activators (see Figure 3) (91). These studies suggest that many of the reported NLRP3 stimuli (such as TLR or NOD ligands) that act in combination with ATP or pore-forming agents are in fact not direct activators of NLRP3. Rather, these stimuli are required for the transcriptional regulation of NLRP3, which at a certain threshold level can then respond to inflammasome activators such as lysosomal damage, ATP, or pore-forming toxins. Thus, the range of NLRP3 activators is likely to be much less broad than previously expected.

The NLRC4 inflammasome. The NLRC4 (also known as IPAF) inflammasome was recently reviewed elsewhere (105), so we will only briefly discuss it here. NLRC4 is the only member of the NLRC family currently known to assemble an inflammasome. With its CARD, NLRC4 can directly recruit caspase- 1 without the need of an adaptor protein (106). However, an interaction with the adaptor protein ASC is still needed for robust IL-1 $\beta$ secretion (107-110). NLRC4-deficient macrophages show markedly reduced secretion of IL-1 $\beta$ and pyroptosis after infection with Salmonella typhimurium, Legionella pneumophila, Shigella flexneri, and Pseudomonas aeru- ginosa $(107,109-114)$. In response to these bacteria, oligomerization of NLRC4 is triggered by cytosolic flagellin or other stimuli possibly delivered by a bacterial secretion system (type III or type IV) $(108,109,112,114,115)$. Some studies see a role for NAIP5, another member of the NLR family, in recognition of the ligand under certain circumstances $(111,116,117)$. Oligomerized NLRC4 associates with pro-caspase- 1 via the CARDs, leading to autocleavage of caspase- $1(106,118,119)$. Active caspase- 1 in turn activates cytokines of the IL-1 family and leads to pyroptosis. Flagellin as a possible ligand offers the intriguing possibility that a single PAMP could trigger both IL-1 $\beta$ priming via TLR5 (120) and maturation via caspase-1. It should be noted that activation of NLRC4 itself in response to flagellin is independent of TLR5 (108).

DNA-sensing by the AIM2 inflammasome. In a series of experiments performed by Tschopp and colleagues (51), infection with adenoviruses triggered macrophages to release IL-1 $\beta$ in an NLRP3- and ASC-dependent pathway. However, upon transfection of E. coli, viral, mammalian, or synthetic DNA [poly(dA:dT)], IL- $1 \beta$ release was triggered in an ASC-dependent, yet NLRP3-independent manner. These experiments suggested the existence of a DNA-sensing, NLRP3-independent inflammasome that requires ASC for the activation of caspase-1. The sensor for double-stranded DNA was iden- 
Table 1

Pathologic activation of NLRs/inflammasomes, and associated diseases

\author{
Disease \\ Hereditary periodic fevers \\ Cryopyrinopathies \\ (MWS, FCAS, NOMID) \\ FMF \\ NLRP12 familial fever
}

Other genetic diseases
Vitiligo
Crystal-induced diseases
Gout
Pseudogout
Asbestosis
Silicosis
Other
Alzheimer disease
Contact hypersensitivity

\author{
Responsible factor \\ Mutation in CIAS1/NLRP3 \\ Mutations in MEFV/pyrin \\ Truncation mutations in NLRP12
}

Mutations in NLRP1

MSU crystals
Calcium pyrophosphate dehydrate
Asbestos
Silica

$\beta$-Amyloid

Trinitrochlorobenzene, sodium dodecylsulfate
Effect

Refs.

Overactive variants of NLRP3, overexpression of NLRP3 leading to facilitated inflammasome activation

Possible promotion of ASC assembly

Hereditary periodic fevers associated with arthralgia, and urticaria

Unknown

Activation of the NLRP3 inflammasome

Activation of the NLRP3 inflammasome

Activation of the NLRP3 inflammasome

Activation of the NLRP3 inflammasome

Activation of the NLRP3 inflammasome

Activation of the NLRP3 inflammasome
128,129

133

137

73

73

75

$74-76$

84

85 tified to be the IFN-inducible protein AIM2 (10-13). AIM2 contains a HIN200 domain, which recognizes double-stranded DNA, and a PYD, which allows for recruitment of ASC and the formation of a caspase-1-activating inflammasome $(11,12)$. AIM2 likely plays a role in innate defense against DNA viruses and intracellular bacteria $(11,12)$. In addition, it may have a function in the recognition of lupus DNA immune complexes and could mediate adjuvanticity in DNA vaccines. The in vivo function of AIM2 should be clarified by the characterization of Aim 2 gene-deficient mice.

Other inflammasomes?The subfamily of NLRPs has 14 members in total. At the time of writing, only two members (NLRP1 and NLRP3) were found to have activating ligands, as described above. NLRP2, NLRP6, and NLRP12 have been proposed to activate caspase- 1 in association with ASC in vitro, but their physiological role remains to be determined (121-123). There are even more proteins with PYDs that might potentially activate caspase- 1 via the adaptor ASC. One member of this family is pyrin (marenostrin), which has also been proposed to assemble a caspase-1-activating inflammasome with ASC $(124,125)$. However, pyrin has also been reported as a negative regulator of ASC assembly (126). Its exact role remains to be established.

\section{Pathologic inflammasome activation}

The importance of inflammasome activation and subsequent IL-1 $\beta$ production in inflammation is underscored by the fact that genetic variants of inflammasome-associated genes play a role in autoinflammatory and autoimmune diseases. Familial hereditary periodic fever syndromes are a group of several distinct autoinflammatory diseases characterized by seemingly unexplained episodes of fever, localized inflammation, and sometimes urticarial rashes (127). Muckle-Wells syndrome (MWS), familial cold autoinflammatory syndrome (FCAS), and neonatal onset multisystem inflammatory disease (NOMID, also known as chronic infantile neurologic cutaneous and arthropathy syndrome) are three phenotypic disease varieties of one single cause: mutations in the CIAS1 gene coding for NLRP3 or possibly in the NLRP3 promotor (128,
129). Some disease-associated NLRP3 variants exhibit increased oligomerization with ASC, thereby facilitating caspase- 1 activation (124). In other cases, mutations in the promoter region of CIAS1 were found, possibly leading to enhanced transcription (130). Animal models using mutant NLRP3 knockin mouse strains show that indeed, the disease phenotype is dependent on the inflammasome, partially dependent on IL-1 $\beta$, and independent of T cells (131). Urticarial rashes might be caused by resident mast cells in the skin, which express inflammasome components and can mediate IL-1 $\beta$ secretion dependent on NLRP3 and ASC (132). Another form of hereditary fever, familial Mediterranean fever (FMF), is caused by mutations in the $M E F V$ gene encoding pyrin (133). As discussed above, it is still unclear whether pyrin inhibits or promotes ASC assembly, but its role in FMF emphasizes its importance for the regulation of caspase-1 activity.

Genetic variants of NLRP1 confer risk not to hereditary fevers, but to generalized vitiligo and associated autoimmune diseases including type 1 diabetes and autoimmune Addison disease (134-136). Generalized vitiligo is a multifactorial disease in which loss of melanocytes results in patchy depigmentation of skin, hair, and mucous membranes.

Mutations in other, less-studied family members of the NLRP family are associated with hereditary fever syndromes, such as in NLRP12 (137). The role of NLRP12 in caspase-1 activation is still unclear. The pathologic activation of NLRs is associated with a variety of diseases (Table 1).

\section{Concluding remarks}

The inflammasomes are rapidly emerging as novel and important cellular complexes involved in the detection of pathogens and endogenous danger signals. They play an important role in immunity, as they are involved in the production of IL-1 $\beta$ and IL- 18 . The receptors for these cytokines activate MyD88 and therefore, like TLRs, inflammasomes activate many pro-inflammatory mediators. There are many challenging open questions, such as whether all of the NLRs are related to one or more inflammasomes, the 


\section{science in medicine}

exact nature of the molecular events leading to inflammasome formation, and the identity of activators for the large number of NLR proteins, especially the pyrin-containing family members. No ligands have yet been identified for NLRP2 and NLRP4-14, but some of these proteins have already been shown to assemble inflammasomes (121-123). The design and discovery of effective and specific drugs that alter inflammasome function have the potential to improve the symptoms of crippling autoinflammatory diseases, crystal-induced inflammation, and possibly an array of other inflammatory conditions not currently thought to be linked to inflammasome activation. As more tools become available, the future of inflammasome research seems particularly exciting.

\section{Acknowledgments}

We would like to acknowledge funding by the NIH (grants AI065483 and AI-083713).

Address correspondence to: Eicke Latz, Department of Infectious Diseases and Immunology, LRB 308, University of Massachusetts Medical School, Worcester, Massachusetts 01605, USA. Phone: (508) 856-5889; Fax: (508) 856-5463; Email: eicke.latz@ umassmed.edu. Alternate address: Eicke Latz, Institute of Innate Immunity, Biomedical Center, Sigmund-Freud-Str. 25, 53127 Bonn, Germany. Phone: 0228-287-51223; Fax: 0228-287-51221; E-mail: eicke.latz@uni-bonn.de.
1. Akahoshi, T., Murakami, Y., and Kitasato, H. 2007. Recent advances in crystal-induced acute inflammation. Curr. Opin. Rheumatol. 19:146-150.

2. Duncan, J.A., et al. 2007. Cryopyrin/NALP3 binds ATP/dATP, is an ATPase, and requires ATP binding to mediate inflammatory signaling. Proc. Natl. Acad. Sci. U. S. A. 104:8041-8046.

3. Ye, Z., et al. 2008. ATP binding by monarch-1/ NLRP12 is critical for its inhibitory function. Mol. Cell. Biol. 28:1841-1850.

4. Ting, J.P.Y., et al. 2008. The NLR gene family: a standard nomenclature. Immunity. 28:285-287.

5. Zou, H., Li, Y., Liu, X., and Wang, X. 1999. An APAF-1.cytochrome $c$ multimeric complex is a functional apoptosome that activates procaspase-9. J. Biol. Chem. 274:11549-11556.

6. Kim, H.E., Du, F., Fang, M., and Wang, X. 2005. Formation of apoptosome is initiated by cytochrome cinduced dATP hydrolysis and subsequent nucleotide exchange on Apaf-1. Proc. Natl. Acad. Sci. U. S. A. 102:17545-17550.

7. Bao, Q., and Shi, Y. 2007. Apoptosome: a platform for the activation of initiator caspases. Cell Death Differ. 14:56-65.

8. Martinon, F., Burns, K., and Tschopp, J. 2002. The inflammasome: a molecular platform triggering activation of inflammatory caspases and processing of proIL-beta. Mol. Cell. 10:417-426.

9. Martinon, F., Mayor, A., and Tschopp, J. 2009. The inflammasomes: guardians of the body. Annu. Rev. Immunol. 27:229-265.

10. Buerckstuemmer, T., et al. 2009. An orthogonal proteomic-genomic screen identifies AIM2 as a cytoplasmic DNA sensor for the inflammasome. Nat. Immunol. 10:266-272.

11. Fernandes-Alnemri, T., Yu, J.-W., Datta, P., Wu, J., and Alnemri, E.S. 2009. AIM2 activates the inflammasome and cell death in response to cytoplasmic DNA. Nature. 458:509-513.

12. Hornung, V., et al. 2009. AIM2 recognizes cytosolic dsDNA and forms a caspase-1-activating inflammasome with ASC. Nature. 458:514-518.

13. Roberts, T.L., et al. 2009. HIN-200 proteins regulate caspase activation in response to foreign cytoplasmic DNA. Science. 323:1057-1060.

14. Thornberry, N.A., et al. 1992. A novel heterodimeric cysteine protease is required for interleukin-1 beta processing in monocytes. Nature. 356:768-774.

15. Wilson, K.P., et al. 1994. Structure and mechanism of interleukin-1 beta converting enzyme. Nature. 370:270-275.

16. Dinarello, C.A. 2009. Immunological and inflammatory functions of the interleukin-1 family. Annu. Rev. Immunol. 27:519-550.

17. Kahlenberg, J.M., Lundberg, K.C., Kertesy, S.B., Qu, Y., and Dubyak, G.R. 2005. Potentiation of caspase-1 activation by the $\mathrm{P} 2 \mathrm{X} 7$ receptor is dependent on TLR signals and requires NF-kappaB-driven protein synthesis. J. Immunol. 175:7611-7622.

18. Gu, Y., et al. 1997. Activation of interferon-gamma inducing factor mediated by interleukin-1beta converting enzyme. Science. 275:206-209.

19. Cayrol, C., and Girard, J.-P. 2009. The IL-1-like cytokine IL-33 is inactivated after maturation by caspase-1. Proc. Natl. Acad. Sci. U. S. A. 106:9021-9026.

20. Talabot-Ayer, D., Lamacchia, C., Gabay, C., and Palmer, G. 2009. Interleukin-33 is biologically active independently of caspase-1 cleavage. J. Biol. Chem.284:19420-19426.

21. Luthi, A.U., et al. 2009. Suppression of interleukin33 bioactivity through proteolysis by apoptotic caspases. Immunity. 31:84-98.

22. Andrei, C., et al. 2004. Phospholipases C and A2 control lysosome-mediated IL-1 beta secretion: Implications for inflammatory processes. Proc. Natl. Acad. Sci. U. S. A. 101:9745-9750.

23. Brough, D., and Rothwell, N.J. 2007. Caspase-1-dependent processing of pro-interleukin-1beta is cytosolic and precedes cell death. J. Cell Sci. 120:772-781.

24. MacKenzie, A., et al. 2001. Rapid secretion of interleukin-1beta by microvesicle shedding. Immunity. 15:825-835.

25. Qu, Y., Franchi, L., Nunez, G., and Dubyak, G.R. 2007. Nonclassical IL-1 beta secretion stimulated by $\mathrm{P} 2 \mathrm{X} 7$ receptors is dependent on inflammasome activation and correlated with exosome release in murine macrophages. J. Immunol. 179:1913-1925.

26. Labbé, K., and Saleh, M. 2008. Cell death in the host response to infection. Cell Death Differ. 15:1339-1349.

27. Ting, J.P., Willingham, S.B., and Bergstralh, D.T. 2008. NLRs at the intersection of cell death and immunity. Nat. Rev. Immunol. 8:372-379.

28. Fink, S.L., and Cookson, B.T. 2006. Caspase-1dependent pore formation during pyroptosis leads to osmotic lysis of infected host macrophages. Cell. Microbiol. 8:1812-1825.

29. Shao, W., Yeretssian, G., Doiron, K., Hussain, S.N., and Saleh, M. 2007. The caspase-1 digestome identifies the glycolysis pathway as a target during infection and septic shock. J. Biol. Chem. 282:36321-36329.

30. Lamkanfi, M., et al. 2008. Targeted peptidecentric proteomics reveals caspase- 7 as a substrate of the caspase-1 inflammasomes. Mol. Cell Proteomics. 7:2350-2363.

31. Gurcel, L., Abrami, L., Girardin, S., Tschopp, J., and van der Goot, F.G. 2006. Caspase-1 activation of lipid metabolic pathways in response to bacterial pore-forming toxins promotes cell survival. Cell. 126:1135-1145.

32. Keller, M., Ruegg, A., Werner, S., and Beer, H.D. 2008. Active caspase- 1 is a regulator of unconventional protein secretion. Cell. 132:818-831.

33. Martinon, F., Hofmann, K., and Tschopp, J. 2001. The pyrin domain: a possible member of the death domain-fold family implicated in apoptosis and inflammation. Curr. Biol. 11:R118-R120.

34. Faustin, B., et al. 2007. Reconstituted NALP1 inflammasome reveals two-step mechanism of caspase-1 activation. Mol. Cell. 25:713-724.

35. Faustin, B., et al. 2009. Mechanism of Bcl-2 and
Bcl-X(L) inhibition of NLRP1 inflammasome: loop domain-dependent suppression of ATP binding and oligomerization. Proc. Natl. Acad. Sci. U. S. A. 106:3935-3940.

36. Bruey, J.M., et al. 2007. Bcl-2 and Bcl-XL regulate proinflammatory caspase- 1 activation by interaction with NALP1. Cell. 129:45-56.

37. Boyden, E.D., and Dietrich, W.F. 2006. Nalp1b controls mouse macrophage susceptibility to anthrax lethal toxin. Nat. Genet. 38:240-244.

38. Fink, S.L., Bergsbaken, T., and Cookson, B.T. 2008. Anthrax lethal toxin and Salmonella elicit the common cell death pathway of caspase-1-dependent pyroptosis via distinct mechanisms. Proc. Natl. Acad. Sci. U. S. A. 105:4312-4317.

39. Wickliffe, K.E., Leppla, S.H., and Moayeri, M. 2008. Anthrax lethal toxin-induced inflammasome formation and caspase- 1 activation are late events dependent on ion fluxes and the proteasome. Cell. Microbiol. 10:332-343.

40. Squires, R.C., Muehlbauer, S.M., and Brojatsch, J. 2007. Proteasomes control caspase- 1 activation in anthrax lethal toxin-mediated cell killing. J. Biol. Chem. 282:34260-34267.

41. Hsu, L.-C., et al. 2008. A NOD2-NALP1 complex mediates caspase-1-dependent IL-1beta secretion in response to Bacillus anthracis infection and muramyl dipeptide. Proc. Natl. Acad. Sci. U. S. A. 105:7803-7808.

42. Ferwerda, G., et al. 2008. Engagement of NOD2 has a dual effect on proIL-1beta mRNA transcription and secretion of bioactive IL-1beta. Eur. J. Immunol. 38:184-191.

43. Girardin, S.E., et al. 2003. Nod2 is a general sensor of peptidoglycan through muramyl dipeptide (MDP) detection. J. Biol. Chem. 278:8869-8872.

44. Ogura, Y., et al. 2001. A frameshift mutation in NOD2 associated with susceptibility to Crohn's disease. Nature. 411:603-606.

45. Manji, G.A., et al. 2002. PYPAF1, a PYRIN-containing Apaf1-like protein that assembles with ASC and regulates activation of NF-kappa B. J. Biol. Chem. 277:11570-11575.

46. Agostini, L., et al. 2004. NALP3 forms an IL-1betaprocessing inflammasome with increased activity in Muckle-Wells autoinflammatory disorder. Immunity. 20:319-325.

47. Duncan, J.A., et al. 2007. Cryopyrin/NALP3 binds ATP/dATP, is an ATPase, and requires ATP binding to mediate inflammatory signaling. Proc. Natl. Acad. Sci. U. S. A. 104:8041-8046.

48. Pétrilli, V., et al. 2007. Activation of the NALP3 inflammasome is triggered by low intracellular potassium concentration. Cell Death Differ. 14:1583-1589.

49. Fernandes-Alnemri, T., et al. 2007. The pyroptosome: a supramolecular assembly of ASC dimers mediating inflammatory cell death via caspase- 1 activation. Cell Death Differ. 14:1590-1604.

50. Kanneganti, T.-D., et al. 2006. Critical role for Cryopyrin/Nalp3 in activation of caspase- 1 in response to viral infection and double-stranded RNA. J. Biol. 
Chem. 281:36560-36568.

51. Muruve, D.A., et al. 2008. The inflammasome recognizes cytosolic microbial and host DNA and triggers an innate immune response. Nature. 452:103-107.

52. Thomas, P.G., et al. 2009. The intracellular sensor NLRP3 mediates key innate and healing responses to influenza A virus via the regulation of caspase- 1 . Immunity. 30:566-575.

53. Allen, I.C., et al. 2009. The NLRP3 inflammasome mediates in vivo innate immunity to influenza a virus through recognition of viral RNA. Immunity. 30:556-565.

54. Koo, I.C., et al. 2008. ESX-1-dependent cytolysis in lysosome secretion and inflammasome activation during mycobacterial infection. Cell. Microbiol. 10:1866-1878

55. Duncan, J.A., et al. 2009. Neisseria gonorrhoeae activates the proteinase cathepsin $\mathrm{B}$ to mediate the signaling activities of the NLRP3 and ASC-containing inflammasome. J. Immunol. 182:6460-6469.

56. Gross, O., et al. 2009. Syk kinase signalling couples to the Nlrp3 inflammasome for anti-fungal host defence. Nature. 459:433-436.

57. van de Veerdonk, F.L., et al. 2009. Bypassing pathogen-induced inflammasome activation for the regulation of interleukin-1beta production by the fungal pathogen Candida albicans. J. Infect. Dis. 199:1087-1096.

58. Hise, A.G., et al. 2009. An essential role for the NLRP3 inflammasome in host defense against the human fungal pathogen Candida albicans. Cell Host Microbe. 5:487-497.

59. Kanneganti, T.-D., et al. 2007. Pannexin-1-mediated recognition of bacterial molecules activates the cryopyrin inflammasome independent of Toll-like receptor signaling. Immunity. 26:433-443.

60. Kanneganti, T.-D., et al. 2006. Bacterial RNA and small antiviral compounds activate caspase- 1 through cryopyrin/Nalp3. Nature. 440:233-236.

61. Mariathasan, S., et al. 2006. Cryopyrin activates the inflammasome in response to toxins and ATP Nature. 440:228-232.

62. Munoz-Planillo, R., Franchi, L., Miller, L.S., and Nunez, G. 2009. A critical role for hemolysins and bacterial lipoproteins in Staphylococcus aureusinduced activation of the Nlrp3 inflammasome. J. Immunol. 183:3942-3948.

63. Pelegrin, P., and Surprenant, A. 2006. Pannexin-1 mediates large pore formation and interleukin1 beta release by the ATP-gated $\mathrm{P} 2 \mathrm{X} 7$ receptor. EMBO J. 25:5071-5082.

64. Pelegrin, P., and Surprenant, A. 2007. Pannexin-1 couples to maitotoxin- and nigericin-induced interleukin-1beta release through a dye uptake-independent pathway. J. Biol. Chem. 282:2386-2394.

65. Locovei, S., Scemes, E., Qiu, F., Spray, D.C., and Dahl, G. 2007. Pannexin1 is part of the pore forming unit of the $\mathrm{P} 2 \mathrm{X}(7)$ receptor death complex. FEBS Lett. 581:483-488.

66. Locovei, S., Wang, J., and Dahl, G. 2006. Activation of pannexin 1 channels by ATP through P2Y receptors and by cytoplasmic calcium. FEBS Lett. 580:239-244.

67. Filippini, A., Taffs, R.E., and Sitkovsky, M.V. 1990. Extracellular ATP in T-lymphocyte activation: possible role in effector functions. Proc. Natl. Acad. Sci. U. S. A. 87:8267-8271.

68. Ferrari, D., Chiozzi, P., Falzoni, S., Hanau, S., and Di Virgilio, F. 1997. Purinergic modulation of interleukin-1 beta release from microglial cells stimulated with bacterial endotoxin. J. Exp. Med. 185:579-582.

69. Piccini, A., et al. 2008. ATP is released by monocytes stimulated with pathogen-sensing receptor ligands and induces IL-1beta and IL-18 secretion in an autocrine way. Proc. Natl. Acad. Sci. U. S. A. 105:8067-8072.

70. Netea, M.G., et al. 2009. Differential requirement for the activation of the inflammasome for processing and release of IL-1beta in monocytes and macrophages. Blood. 113:2324-2335.

71. Yamasaki, K., et al. 2009. NLRP3/cryopyrin is necessary for interleukin-1\{beta\} (IL-1\{beta\}) release in response to hyaluronan, an endogenous trigger of inflammation in response to injury. J. Biol. Chem. 284:12762-12771.

72. McCarty, D.J. 1974. Crystal deposition joint disease. Annu. Rev. Med. 25:279-288.

73. Martinon, F., Pétrilli, V., Mayor, A., Tardivel, A., and Tschopp, J. 2006. Gout-associated uric acid crystals activate the NALP3 inflammasome. Nature. 440:237-241

74. Hornung, V., et al. 2008. Silica crystals and aluminum salts activate the NALP3 inflammasome through phagosomal destabilization. Nat. Immunol. 9:847-856.

75. Dostert, C., et al. 2008. Innate immune activation through Nalp3 inflammasome sensing of asbestos and silica. Science. 320:674-677.

76. Cassel, S.L., et al. 2008. The Nalp3 inflammasome is essential for the development of silicosis. Proc. Natl. Acad. Sci. U. S. A. 105:9035-9040.

77. Demento, S.L., et al. 2009. Inflammasome-activating nanoparticles as modular systems for optimizing vaccine efficacy. Vaccine. 27:3013-3021.

78. Kool, M., et al. 2008. Cutting edge: alum adjuvant stimulates inflammatory dendritic cells through activation of the NALP3 inflammasome. J. Immu nol. 181:3755-3759.

79. Li, H., Willingham, S.B., Ting, J.P., and Re, F. 2008. Cutting edge: inflammasome activation by alum and alum's adjuvant effect are mediated by NLRP3 J. Immunol. 181:17-21.

80. Eisenbarth, S.C., Colegio, O.R., O'Connor, W., Sutterwala, F.S., and Flavell, R.A. 2008. Crucial role for the Nalp3 inflammasome in the immunostimulatory properties of aluminium adjuvants. Nature. 453:1122-1126.

81. Franchi, L., and Nunez, G. 2008. The Nlrp3 inflammasome is critical for aluminium hydroxide-mediated IL-1beta secretion but dispensable for adjuvant activity. Eur. J. Immunol. 38:2085-2089.

82. Sharp, F.A., et al. 2009. Uptake of particulate vaccine adjuvants by dendritic cells activates the NALP3 inflammasome. Proc. Natl. Acad. Sci. U. S. A. 106:870-875

83. Dostert, C., et al. 2009. Malarial hemozoin is a Nalp3 inflammasome activating danger signal. PLoS One. 4:e6510.

84. Halle, A., et al. 2008. The NALP3 inflammasome is involved in the innate immune response to amyloid-beta. Nat. Immunol. 9:857-865.

85. Watanabe, H., et al. 2007. Activation of the IL-1betaprocessing inflammasome is involved in contact hypersensitivity. J. Invest. Dermatol. 127:1956-1963.

86. Sutterwala, F.S., et al. 2006. Critical role for NALP3/CIAS1/Cryopyrin in innate and adaptive immunity through its regulation of caspase- 1 . Immunity. 24:317-327.

87. Feldmeyer, L., et al. 2007. The inflammasome mediates UVB-induced activation and secretion of interleukin-1beta by keratinocytes. Curr. Biol. 17:1140-1145.

88. Walev, I., Reske, K., Palmer, M., Valeva, A., and Bhakdi, S. 1995. Potassium-inhibited processing of IL-1 beta in human monocytes. EMBO J. 14:1607-1614.

89. Perregaux, D., and Gabel, C.A. 1994. Interleukin-1 beta maturation and release in response to ATP and nigericin. Evidence that potassium depletion mediated by these agents is a necessary and common feature of their activity. J. Biol. Chem. 269:15195-15203.

90. Colomar, A., et al. 2003. Maturation and release of interleukin-1beta by lipopolysaccharide-primed mouse Schwann cells require the stimulation of P2X7 receptors. J. Biol. Chem. 278:30732-30740.
91. Bauernfeind, F.G., et al. 2009. Cutting edge: NFkappaB activating pattern recognition and cytokine receptors license NLRP3 inflammasome activation by regulating NLRP3 expression. J. Immunol. 183:787-791.

92. Franchi, L., Eigenbrod, T., and Nunez, G. 2009. Cutting edge: TNF-alpha mediates sensitization to ATP and silica via the NLRP3 inflammasome in the absence of microbial stimulation. J. Immunol. 183:792-796.

93. Riedl, S.J., and Salvesen, G.S. 2007. The apoptosome: signalling platform of cell death. Nat. Rev. Mol. Cell. Biol. 8:405-413.

94. Cain, K., Langlais, C., Sun, X.M., Brown, D.G., and Cohen, G.M. 2001. Physiological concentrations of $\mathrm{K}+$ inhibit cytochrome c-dependent formation of the apoptosome. J. Biol. Chem. 276:41985-41990.

95. Marina-García, N., et al. 2008. Pannexin-1-mediated intracellular delivery of muramyl dipeptide induces caspase-1 activation via cryopyrin/NLRP3 independently of Nod2. J. Immunol. 180:4050-4057.

96. Cruz, C.M., et al. 2007. ATP activates a reactive oxygen species-dependent oxidative stress response and secretion of proinflammatory cytokines in macrophages. J. Biol. Chem. 282:2871-2879.

97. Kuijk, L.M., et al. 2008. HMG-CoA reductase inhibition induces IL-1beta release through Rac1/ PI3K/PKB-dependent caspase-1 activation. Blood. 112:3563-3573.

98. Meissner, F., Molawi, K., and Zychlinsky, A. 2008. Superoxide dismutase 1 regulates caspase- 1 and endotoxic shock. Nat. Immunol. 9:866-872.

99. Hentze, H., Lin, X.Y., Choi, M.S.K., and Porter, A.G. 2003. Critical role for cathepsin B in mediating caspase-1-dependent interleukin-18 maturation and caspase-1-independent necrosis triggered by the microbial toxin nigericin. Cell Death Differ. 10:956-968.

100.Newman, Z.L., Leppla, S.H., and Moayeri, M. 2009. CA-074Me protection against anthrax lethal toxin. Infect. Immun. 77:4327-4336.

101.Antunes, F., Cadenas, E., and Brunk, U.T. 2001. Apoptosis induced by exposure to a low steadystate concentration of $\mathrm{H} 2 \mathrm{O} 2$ is a consequence of lysosomal rupture. Biochem. J. 356:549-555.

102.Roberg, K., Johansson, U., and Ollinger, K. 1999. Lysosomal release of cathepsin D precedes relocation of cytochrome $\mathrm{c}$ and loss of mitochondrial transmembrane potential during apoptosis induced by oxidative stress. Free Radic. Biol. Med. 27:1228-1237.

103. Roberg, K., and Ollinger, K. 1998. Oxidative stress causes relocation of the lysosomal enzyme cathep$\sin \mathrm{D}$ with ensuing apoptosis in neonatal rat cardiomyocytes. Am. J. Pathol. 152:1151-1156.

104.Kagedal, K., Johansson, U., and Ollinger, K. 2001. The lysosomal protease cathepsin D mediates apoptosis induced by oxidative stress. FASEB J. 15:1592-1594.

105.Sutterwala, F.S., and Flavell, R.A. 2009. NLRC4/ IPAF: a CARD carrying member of the NLR family. Clin. Immunol. 130:2-6.

106.Geddes, B.J., et al. 2001. Human CARD12 is a novel CED4/Apaf-1 family member that induces apoptosis. Biochem. Biophys. Res. Commun. 284:77-82.

107. Mariathasan, S., et al. 2004. Differential activation of the inflammasome by caspase-1 adaptors ASC and Ipaf. Nature. 430:213-218.

108. Franchi, L., et al. 2006. Cytosolic flagellin requires Ipaf for activation of caspase- 1 and interleukin 1beta in salmonella-infected macrophages. Nat. Immunol. 7:576-582.

109. Miao, E.A., et al. 2006. Cytoplasmic flagellin activates caspase- 1 and secretion of interleukin 1beta via Ipaf. Nat. Immunol. 7:569-575.

110. Sutterwala, F.S., et al. 2007. Immune recognition of Pseudomonas aeruginosa mediated by the IPAF/ NLRC4 inflammasome. J. Exp. Med. 204:3235-3245. 
111.Zamboni, D.S., et al. 2006. The Birc1e cytosolic pattern-recognition receptor contributes to the detection and control of Legionella pneumophila infection. Nat. Immunol. 7:318-325.

112.Suzuki, T., et al. 2007. Differential regulation of caspase- 1 activation, pyroptosis, and autophagy via Ipaf and ASC in Shigella-infected macrophages. PLoS Pathog. 3:e111.

113.Miao, E.A., Ernst, R.K., Dors, M., Mao, D.P., and Aderem, A. 2008. Pseudomonas aeruginosa activates caspase 1 through Ipaf. Proc. Natl. Acad. Sci. U. S. A. 105:2562-2567.

114.Franchi, L., et al. 2007. Critical role for Ipaf in Pseudomonas aeruginosa-induced caspase- 1 activation. Eur. J. Immunol. 37:3030-3039.

115.Sun, Y.-H., Rolán, H.G., and Tsolis, R.M. 2007. Injection of flagellin into the host cell cytosol by Salmonella enterica serotype Typhimurium. J. Biol. Chem. 282:33897-33901.

116. Lamkanfi, M., et al. 2007. The Nod-like receptor family member Naip5/Birc1e restricts Legionella pneumophila growth independently of caspase-1 activation. J. Immunol. 178:8022-8027.

117. Lightfield, K.L., et al. 2008. Critical function for Naip5 in inflammasome activation by a conserved carboxy-terminal domain of flagellin. Nat. Immu nol. 9:1171-1178.

118. Poyet, J.L., et al. 2001. Identification of Ipaf, a human caspase-1-activating protein related to Apaf-1.J. Biol. Chem. 276:28309-28313.

119.Damiano, J.S., Stehlik, C., Pio, F., Godzik, A., and Reed, J.C. 2001. CLAN, a novel human CED-4-like gene. Genomics. 75:77-83.

120. Hayashi, F., et al. 2001. The innate immune response to bacterial flagellin is mediated by Tolllike receptor 5. Nature. 410:1099-1103.

121.Wang, L., et al. 2002. PYPAF7, a novel PYRIN-containing Apaf1-like protein that regulates activation of NF-kappa B and caspase-1-dependent cytokine processing. J. Biol. Chem. 277:29874-29880.

122. Grenier, J.M., et al. 2002. Functional screening of five PYPAF family members identifies PYPAF5 as a novel regulator of NF-kappaB and caspase-1. FEBS Lett. 530:73-78.

123.Srinivasula, S.M., et al. 2002. The PYRIN-CARD protein ASC is an activating adaptor for caspase-1. J. Biol. Chem. 277:21119-21122.

124.Yu, J.W., et al. 2006. Cryopyrin and pyrin activate caspase-1, but not NF-kappaB, via ASC oligomerization. Cell Death Differ. 13:236-249.

125.Yu, J.-W., et al. 2007. Pyrin activates the ASC pyroptosome in response to engagement by autoinflammatory PSTPIP1 mutants. Mol. Cell. 28:214-227.

126. Papin, S., et al. 2007. The SPRY domain of Pyrin, mutated in familial Mediterranean fever patients, interacts with inflammasome components and inhibits proIL-1beta processing. Cell Death Differ. 14:1457-1466.

127. Masters, S.L., Simon, A., Aksentijevich, I., and Kastner, D.L. 2009. Horror autoinflammaticus: the molecular pathophysiology of autoinflammatory disease. Annu. Rev. Immunol. 27:621-668.

128. Hoffman, H.M., Mueller, J.L., Broide, D.H., Wanderer, A.A., and Kolodner, R.D. 2001. Mutation of a new gene encoding a putative pyrin-like protein causes familial cold autoinflammatory syndrome and Muckle-Wells syndrome. Nat. Genet. 29:301-305.

129. Feldmann, J., et al. 2002. Chronic infantile neurological cutaneous and articular syndrome is caused by mutations in CIAS1, a gene highly expressed in polymorphonuclear cells and chondrocytes. Am. J. Hum. Genet. 71:198-203.

130. Anderson, J.P., et al. 2008. Initial description of the human NLRP3 promoter. Genes Immun. 9:721-726.

131. Brydges, S.D., et al. 2009. Inflammasome-mediated disease animal models reveal roles for innate but not adaptive immunity. Immunity. 30:875-887.

132. Nakamura, Y., et al. 2009. Mast cells mediate neutrophil recruitment and vascular leakage through the NLRP3 inflammasome in histamine-independent urticaria. J. Exp. Med. 206:1037-1046.

133. Bernot, A., et al. 1997. A candidate gene for familial Mediterranean fever. Nat. Genet. 17:25-31.

134. Magitta, N.F., et al. 2009. A coding polymorphism in NALP1 confers risk for autoimmune Addison's disease and type 1 diabetes. Genes Immun. 10:120-124.

135.Jin, Y., et al. 2007. NALP1 in vitiligo-associated multiple autoimmune disease. N. Engl. J. Med. 356:1216-1225.

136.Jin, Y., Birlea, S.A., Fain, P.R., and Spritz, R.A. 2007. Genetic variations in NALP1 are associated with generalized vitiligo in a Romanian population. J. Invest. Dermatol. 127:2558-2562.

137.Jeru, I., et al. 2008. Mutations in NALP12 cause hereditary periodic fever syndromes. Proc. Natl. Acad. Sci. U. S. A. 105:1614-1619. 